tion-site skin disinfection. Use of chlorhexidine gluconate is a simple and effective means of reducing vascular catheter-related infections.

FROM: Chaiyakunapruk N, Veenstra DL, Lipsky BA, Saint S. Chlorhexidine compared with povidone-iodine solution for vascular catheter-site care: a meta-analysis. Ann Intern Med 2002;136:792-801.

\section{Nasal Myiasis Linked to Hospital-Wide Mouse Infestation}

Beckendorf and colleagues from the University of Arizona Health Sciences Center reported a large city hospital experience with infestation of mice combated in part by broadcasting poisoned baits. Months later there was an invasion of flies into the hospital, and two comatose patients in an intensive care unit contracted nasal maggots. Adult flies were trapped and maggots removed from the nares of the second patient. These were identified as the green blowfly (Phaenicia sericata).

Recent downsizing of hospital personnel had led to the unintended and unrecognized loss of housekeeping services in the canteen food storage areas. A mouse infestation of the hospital occurred, with the epicenter in the canteen area. This was initially addressed by scattering poisoned bait and using rodent glue boards. The result of such treatment was the presence of numerous mouse carcasses scattered throughout the building attracting the green blowfly. Adult gravid female flies trapped in the new intensive care unit (where mice were not present) laid eggs in the fetid nasal discharge of two comatose patients. Live trapping of mice and removal of carcasses led to an abatement of the fly infestation. The cause-and-effect nature of the mouse carcasses and flies was underscored a year later when an outbreak of $P$. sericata occurred in the operating department and was linked to the presence of mouse carcasses on glue boards not removed the previous fall. Hence, the disruption or loss of one vital link in hospital organization (in this case, housekeeping support) may lead to an unintended and bizarre outcome.

FROM: Beckendorf $\mathrm{R}$, Klotz SA, Hinkle $\mathrm{N}$, Bartholomew W. Nasal myiasis in an intensive care unit linked to hospital-wide mouse infestation. Arch Intern Med 2002;162:638-640.

\section{Vancomycin-Resistant Enterococci in Pork and Poultry Products in France}

Gambarotto and co-investigators from the Limoges University Teaching Hospital, Limoges, France, collected meat products from public retail outlets and tested them for the presence of vancomycin-resistant enterococci (VRE) in an area with a high prevalence of VRE reported in human fecal samples. VRE were detected in $66 \%$ of the samples, and a predominance of VanC strains was found, which is also true for human fecal samples.

FROM: Gambarotto K, Ploy MC, Dupron F, Giangiobbe M, Denis F. Occurrence of vancomycin-resistant enterococci in pork and poultry products from a cattle-rearing area of France. J Clin Microbiol 2001;39:23542355.

\section{Risk of Hepatitis C Virus Transmission From Surgeon to Patient: A 7-Year Retrospective Investigation}

Currently, it is not known how often hepatitis $\mathrm{C}$ virus (HCV) is transmitted from infected healthcare workers to patients during medical care. Ross and colleagues from the Institute of Virology, National Reference Centre for Hepatitis C, Essen, Germany, tried to determine the rate of provider-to-patient transmission of $\mathrm{HCV}$ among former patients of an $\mathrm{HCV}$-positive gynecologist after it was proven that he had infected one of his patients with $\mathrm{HCV}$ during a cesarean section.

All 2,907 women who had been operated on by the HCV-positive gynecologist between July 1993 and March 2000 were notified about potential exposure and were offered free counseling and testing. The crucial differentiation between $\mathrm{HCV}$ transmissions caused by the gynecologist and infections contracted from other sources was achieved by epidemiologic investigations, nucleotide sequencing, and phylogenetic analysis.

Of the 2,907 women affected, $78.6 \%$ could be screened for markers of $\mathrm{HCV}$ infection. Seven of these former patients were found to have HCV. Phylogenetic analysis of $\mathrm{HCV}$ sequences from the gynecologist and the women did not indicate that the virus strains were linked. Therefore, no further iatrogenic $\mathrm{HCV}$ infections caused by the gynecologist could be detected. The resulting overall $\mathrm{HCV}$ transmission rate was $0.04 \%$ ( 1 per 2,286; 95\% confidence interval, $0.008 \%$ to $0.25 \%$ ).

The authors concluded that this was the largest retrospective investigation of the risk of provider-to-patient transmission of HCV conducted so far. They noted that their findings support the notion that such transmissions are relatively rare events and might provide a basis for future recommendations on the management of HCVinfected healthcare workers.

FROM: Ross RS, Viazov S, Thormahlen M, et al. Risk of hepatitis $\mathrm{C}$ virus transmission from an infected gynecologist to patients: results of a 7-year retrospective investigation. Arch Intern Med 2002;162:805-810. 